\title{
Mutagenic, Genotoxic and Sub Chronic Oral Safety Analysis of Boswellia Serrata Extract (Boswellin ${ }^{\circledR}$ Super)
}

\author{
Muhammed Majeed ${ }^{1}$, Sankaran Natarajan ${ }^{1}$, Sarang Bani ${ }^{1}$, Anjali Pandey ${ }^{1 *}$, Prakriti Neupane ${ }^{2}$ \\ ${ }^{I}$ Sami Labs Limited, \#19/1, 19/2, I Main, II Phase, Peenya Industrial Area, Bangalore 560 058, \\ Karnataka, India \\ ${ }^{2}$ Clin World Private Limited, \#19/1, 19/2, I Main, II Phase, Peenya Industrial Area, Bangalore 560 \\ 058, Karnataka, India
}

*Corresponding Author: Anjali Pandey, Sami Labs Limited, \#19/1, 19/2, I Main, II Phase, Peenya Industrial Area, Bangalore 560 058, Karnataka, India

\begin{abstract}
Boswellia serrata is a branching tree found abundantly in India, Boswellic acids extracted from resin of plant is used in inflammatory conditions, arthritis and hyperlipidaemia. However, any supplement used for long term use needs to be tested for potential mutagenicity and toxicity. Thus, we assessed the mutagenicity of Boswellia serrata extract by Mammalian Bone Marrow Chromosome Aberration Test and Mammalian Bone Marrow Micronucleus Test and in vitro Bacterial Reverse Mutation Test. Further we assessed in vivo toxicity by acute toxicity study and 90 days sub chronic toxicity study of Boswellia serrata extract. None of the mutagenic assays showed any increase in mutagenicity above background. Also, the acute oral toxicity and sub chronic toxicity study revealed that Boswellia serrata extract was not lethal to animals up to $2000 \mathrm{mg} / \mathrm{kg} /$ day when observed for 14 days and $600 \mathrm{mg} / \mathrm{kg} /$ day when observed for 90 days. Therefore, these data provide evidence that the Boswellia tested have no cytotoxic potency and are not mutagenic. Thus, our findings contribute to the risk assessment of preparations containing Boswellia serrata extract.
\end{abstract}

Keywords: Boswellia serrata extract, Mutagenicity, toxicity, Ames test, genotoxicity

\section{INTRODUCTION}

In Ayurvedic system of medicine, Boswellia serrata is one of the ancient and most appreciated herbs ${ }^{1}$. Boswellia serrata is a branching tree found abundantly in the dry regions of India, Africa and Middle East. Traditionally resin which is available under the bark of the tree, is used in various religious and socio-cultural activities as incense and also in various therapeutic purposes ${ }^{2-3}$. Resin, gum, essential oils and terpenoids are the reported chemical constituents of the exudate ${ }^{4}$. Boswellic acid, medicinally important terpenoids makes up $25-35 \%$ of the resin ${ }^{5-9}$. Boswellic acids are pentacyclic triterpenes, which may exist in an aconfiguration (geminal methyl groups at C-20) or a bb configuration (vicinal methyl groups at C-19/C-20). Furthermore, boswellic acids may contain an oxo-moiety at $\mathrm{C}-11$ and an acetyl moiety at the $\mathrm{C}-3 \mathrm{OH}$ group ${ }^{10}$.

Boswellia serrata extract (BSE) has been stated to have analgesic and psychopharmacological effect from the non-phenolic extract and the alcohol extracted portion is effective in management of inflammatory conditions, arthritis and hyperlipidaemia ${ }^{11}$.The major effect of the BSE is attributed by two boswellic acids namely 11 -keto- $\beta$-boswellic acid (KBA) and acetyl-11-keto- $\beta$-boswellic acid (AKBA). The European Pharmacopoiea 6.0 includes these two boswellic acids as markers to confirm the quality of the air-dried gum resin exudate of $B$. serrata $^{12}$.Further, $\beta$-boswellic acid, 11-keto- $\beta$ boswellic acid and acetyl-11-keto- $\beta$-boswellic acid have been used in apoptosis of tumor cells, specifically affected by leukemia or colon cancer ${ }^{6}$.

Though there are several studies on efficacy of Boswellia serrata on various indications, safety studies of the extract are very few or inadequate. Also, Boswellia serrata extract is usually intended for long term use so potential cytotoxic, genotoxic or mutagenic activity testing of the extract is important. Further, mutagenicity tests help to reduce the genetic or carcinogenetic hazards to humans as several studies showed the connection between mutagenicity and carcinogenicity ${ }^{13}$.In the present study, we have conducted the in vivo acute toxicity study, 90 days sub chronic study, Mammalian Bone Marrow Chromosome Aberration Test and Mammalian Bone Marrow 
Micronucleus Test and invitro Bacterial Reverse Mutation Test of Boswellia serrata extract.

\section{MATERIALS AND METHODS}

\section{Chemicals}

Boswellia serrata extract(Boswellin ${ }^{\circledR}$ Super) is a standardized extract from the gum resin of $B$ serrata enriched to contain boswellic acids namely $\beta$-Boswellic Acid, Acetyl- $\beta$-Boswellic Acid, 11-keto- $\beta$-Boswellic Acid and Acetyl11-keto- $\beta$-Boswellic Acid". It was manufactured and supplied by Sami Labs limited Bangalore, India. Salmonella typhimurium tester strains TA98, TA100, TA1537, TA1535 and TA102 were obtained from Molecular Toxicology, Inc.157, Industrial Park, Dr. Boone, NC 28607.

\section{Animals}

All animals used for the tests following described were healthy adult. For the mammalian bone marrow chromosome aberration test were used 12 mice and for the mammalian bone marrow micronucleus test were used 25 mice. For the acute oral toxicity study were used 12 female Wistar rats. For the dose range finding and main study of the 90 days sub-chronic study were used 28 Wistar rats of either sex and 55 Wistar rats of either sex. Female animals used were nulliparous and non-pregnant.Standard propylene cage with stainless grill having pelleted food and drinking water supply were used to house the animals, three animals per cage in air-conditioned room with adequate fresh air supply (more than 12 air changes per hour) and autoclaved corn cob beddings. The room temperature was maintained in between $19.60^{\circ} \mathrm{C}$ to $23.70^{\circ} \mathrm{C}$ and relative humidity of $50.30-65.60 \%$ with $12 \mathrm{~h}$ light and $12 \mathrm{~h}$ dark cycle during acclimatization, experimental period and recovery period. All animals were provided conventional laboratory rodent diet manufactured by Nutrivet Life Sciences, Pune and purified water was provided ad libitum.

\section{Ethics}

The institutional animal ethics committee (IAEC) had approved the protocol and animals used in the individual protocols bearing approval number sa-FORD_MAY_2015_21_ 004, FORD_Sep_2015_23_037, sa-FORD_ JAN_2015_20_060 and sa-FORD_JAN_2015 _20_063 respectively for acute oral toxicity, 90 days sub-chronic study, Mammalian Bone Marrow Chromosome Aberration Test and mammalian bone marrow micronucleus test respectively. The study was conducted in the CPSEA approved laboratory (reg. No. 1256/ bc/09/CPCSEA) in compliance of CPSEA guidelines and OECD guidelines for testing of chemicals 423, 408, 475, 474 and 471 respectively ${ }^{14-18}$.

\section{Acute Oral Toxicity Study}

The vehicle ( $0.5 \%$ Carboxy methyl cellulose) was added to the weighed quantities of the test item (300 and $2000 \mathrm{mg}$ ) slowly and mixed well to make the formulations. It was then transferred to calibrated measuring cylinders. The stock concentrations of 30 and $200 \mathrm{mg} / \mathrm{ml}$ were achieved by making up the volume to $10 \mathrm{ml}$ in the measuring cylinders. Formulation of the test item was prepared fresh and unused volume was discarded.

The test item was administered orally by gavage as single dose using gavage needle number 14 . Dose was adjusted according to the body weight which was measured on the dosing day and maximum dose volume was $10 \mathrm{ml} / \mathrm{kg}$. Food was given 3-5 hrs after dosing.

The dose given was $300 \mathrm{mg} / \mathrm{kg}$ body weight, $2000 \mathrm{mg} / \mathrm{kg}$ body weight and $2000 \mathrm{mg} / \mathrm{kg}$ body weight in dose range finding study(G1) step I, step II and main study(step III) respectively. All the animals were observed for clinical signs of toxicity and mortality at $30 \mathrm{~min}, 1 \mathrm{hr}, 2 \mathrm{hr}, 3 \mathrm{hr}$ and $4 \mathrm{hr}$ on the day one after dosing and thereafter once daily for clinical signs and twice daily for mortality during the 14 days study period. All the test animals were weighed on day before the dose administration day 0 and on day 7 and 14 during the study period.

\section{Days Sulb Chronic Toxicity Study}

Five rats per sex per group were assigned to the main study group and five rats per sex were assigned for recovery study group after minimum 6 days of acclimatization. Rats were administered with dose of $0,100,200,400$ and $600 \mathrm{mg} / \mathrm{kg}$ body weight/day of test item to Control / control recovery (G1/G1R), Low dose (G2), Mid dose (G3) and High dose / High dose recovery $(\mathrm{G} 4 / \mathrm{G} 4 \mathrm{R})$ groups respectively in both dose range finding study and main study.

All animals were observed twice daily (morning and evening) for morbidity and mortality, throughout the acclimatization and study periods for both Dose Range Finding Study (DRFS) and Main Study (DRMS). Clinical signs of animals were observed before and after dose administration on treatment day 1 and once daily thereafter preferably at the same time each day throughout the experimental and recovery period. Detailed clinical examination was conducted on day 1 , day 7 and day 14 for dose range finding study and was subjected on day 1 
and weekly thereafter for main study. Ophthalmological examination was conducted prior to exposure for all the animals and last week of treatment for control and high dose group in dose range finding study. Whereas in main study it was conducted prior to exposure for all animals and on week $13^{\text {th }}$ for main group and week $17^{\text {th }}$ for recovery group. For main study, functional observation battery/ neurobehavioral observation, sensory reactivity to stimuli, assessment of grip strength, hind limb foot splay and motor activity assessment were performed in week 13th for main group animals and in week 17th for recovery group animals. Body weights were measured at start of treatment, Day 7 and Day 14 and for Main Study, animals were weighed during randomization, at start of treatment and weekly thereafter, till the end of experimental period. Feed Consumption of all animals were determined on Day 7 and Day 14 for Dose Range Finding Study and weekly once, during the treatment and recovery period for Main Study Clinical Pathology evaluation of all surviving rats were performed on the termination day, just prior to necropsy. Blood was collected from retro orbital plexuses in vials without anticoagulant for Clinical Biochemistry, $4.0 \%$ EDTA for Haematology and 3.8\% Sodium Citrate for Coagulation factors just prior to or as part of the procedure for euthanasia of the animals. Clinical Pathological evaluations of blood and serum were done as soon as possible, after collection.

Urine sample was collected early morning before blood sampling on the day of sacrifice. Color, appearance and urine volume were recorded by visual observation and other parameters (blood/blood cell, bilirubin, Glucose, Ketone, Leucocytes, nitrites, protein and $\mathrm{pH}$ ) were analysed using Auto Analyzer.

Complete internal and external examination was done for animals and results were recorded. Histopathological examination was carried out on the preserved organs of control and high dose groups rats.

\section{Mammalian Bone Marrow Chromosome Aberration Test}

The mammalian in vivo chromosome aberration test is used for the detection of structural chromosome aberrations induced by test compounds in bone marrow cells of animals, usually rodents. A DRFS was conducted using four groups vehicle (consisting of three male and three female animals per group); and three treatment groups. Vehicle control group was administered orally with $0.5 \%$ (w/v) carboxy methylcellulose. The treatment groups treated at the dose levels of 500, 1000 and $2000 \mathrm{mg}$ of Boswellia serrata Extract / $\mathrm{kg}$ body weight as low, mid and high dose, respectively, via oral route for two days treatments separated by approximately $24 \mathrm{~h}$. Animals were administered oral gavage with the test item or vehicle using cannula. Positive control was administered by intraperitoneal route. The dose volume used was $10 \mathrm{ml} / \mathrm{kg}$ body weight. Cyclophosphamide monohydrate was used as positive control at 50 $\mathrm{mg} / \mathrm{kg}$ body weight dose level once on the day of second dosing. Colchicine was injected to all the animals intraperitoneally after final treatment at dose level of $4 \mathrm{mg} / \mathrm{kg}$ body weight $2-3$ hours prior to sacrifice.

Samples of bone marrow were taken from both the femurs between 22 - 24 hours following final treatment. The cell suspension was centrifuged at $1000 \mathrm{rpm}$ for $10 \mathrm{~min}$ and the supernatant was discarded. The pelleted cells were re-suspended in approximately $5 \mathrm{~mL}$ hypotonic solution $(0.075 \mathrm{M} \mathrm{KCl})$ and incubated at $37 \pm 2{ }^{\circ} \mathrm{C}$ for 20 minutes.

After exposure to hypotonic solution, the cells were centrifuged at $1000 \mathrm{rpm}$ for 10 minutes at room temperature and supernatant was discarded. The residual cell pellet was mixed with chilled Carnoy's fixative for fixation of cells. The cells were centrifuged again at 1000 rpm for 10 minutes at room temperature and supernatant was discarded. This fixation procedure was repeated 2 times.

After centrifugation, this cell suspension was dropped on a clean chilled slide by hanging drop method and kept for air-drying. Two slides were prepared from each animal. After drying, the slides were stained with $5 \%$ Giemsa stain and mounted with DPX mount.

The Mitotic index was determined as-number of dividing cells per 1,000 cells scored. Except for range finding study, all slides were independently coded before microscopic analysis. The slides were scored for gaps, breaks, fragments, deletions, exchanges and chromosomal disintegrations as structural chromosome aberrations and 100 well spread metaphases per animal were scored for chromosomal damage on coded slides. The number of chromosome aberration per metaphase was determined.

The individual mouse was observed daily during the acclimatization period. With special attention on the day of dosing (day 0) the examinations were made during the first 30 
minutes continuously to assess the onset and determination of adverse effects and at approximately 1, 2 and 4 hours after administration. Body weight of the individual animal was observed and recorded on day 0 , day 1 prior to dosing and on the day of bone marrow collection.

The test item can be considered as positive if (i) There is dose-related increase in the relative number of cells with chromosome aberrations; (ii) There is a clear increase in the number of cells with aberrations in a single dose group at a single sampling time. The test item can be considered as negative if it does not meet the above criteria.

\section{Mammalian Bone Marrow Micronucleus Test}

The purpose of the micronucleus test is to identify substances (liquid or solid) that cause cytogenetic damage which results in the formation of micronuclei containing lagging chromosome fragments or whole chromosomes. After one-week acclimatization, a dose-finding test was conducted using each of 12 male and female mice given Boswellia serrata extract (each 3 mice for three dose groups of 0,500 , 1000 , or $2000 \mathrm{mg} / \mathrm{kg}$ bw/day, orally once a day) for 4 days. Boswellia serrata extract was administered once daily for two days by gavage to 25 male mice (each 5 mice for $0,500,1000$, $2000 \mathrm{mg} / \mathrm{kg}$ and a positive control) for the micronucleus test. MMC ( $2 \mathrm{mg} / \mathrm{kg}$ bw/day in normal saline, only once on the final day of drug administration) was administered by intraperitoneal injection as a positive control.

A needle with syringe filled with fetal bovine serum was inserted through the opening at the lower end of the femur bone to collect bone marrow into the prelabelled tubes. The cell suspension was centrifuged at approximately $1000 \mathrm{rpm}$ for 10 minutes and the supernatant was discarded. A small drop of the resuspended cell pellet was smeared on a prelabelled slide and from each animal two slides were prepared. The smeared slides were fixed in absolute methanol for $5 \mathrm{~min}$ and allowed to air dry for 15 - $20 \mathrm{~min}$. All the slides were stained with Giemsa and May- Grunwald stain.

The proportion of immature among total erythrocytes was determined for each animal by counting a total of at least 200 erythrocytes. Except for range finding study, all slides were independently coded before microscopic analysis. At least 2000 erythrocytes per animal were scored under 100X magnification for the incidence of micronucleated immature erythrocytes.

\section{Bacterial Reverse Mutation Test}

Bacterial cell viability was determined by colony forming units and the tester strain attaining at least $1 \times 10^{9}$ bacterial cell $/ \mathrm{ml}$ were used in the assay. Based on the solubility test, Dimethyl sulfoxide was selected as a vehicle for the test item in the study.

Five concentrations of test item were tested in triplicate plates along with vehicle and positive control. Since, there was no reduction in number of revertant colonies and background lawn in cytotoxicity assay up to the concentration 5000 $\mu \mathrm{g} / \mathrm{plate}$, the revertant count of TA98 and TA100 was directly incorporated from cytotoxicity assay to Trial I for the tested concentration from 312.5 to $5000 \mu \mathrm{g} /$ plate vehicle and positive controls in both presence and absence of metabolic activation.

In Trial II, the assay was performed to confirm the negative results. The tester strains TA1535, TA1537, TA98, TA100 and TA102 were tested only in the presence of S9 metabolic activation system $(10 \%$ v/v S9 mix $)$ using plate incorporation method. Five concentrations of test item were tested in triplicate plates along with vehicle and positive controls.

The reference mutagens used as positive controls in each experiment with and without metabolic activation were as per Table 1:

Table1. Concurrent positive controls included in the study

\begin{tabular}{|c|c|c|c|c|c|}
\hline Positive Control & CAS No. & Vehicle & $\begin{array}{c}\text { Metabolic } \\
\text { Activation }\end{array}$ & Strain & $\begin{array}{c}\text { Concentration } \\
(\boldsymbol{\mu g} / \mathbf{p l a t e})\end{array}$ \\
\hline Sodium azide & $26628-22-8$ & Distilled water & Absence & TA100, TA1535 & 20 \\
\hline 2-Nitrofluorene & $607-57-8$ & Dimethyl sulfoxide & Absence & TA98 & 25 \\
\hline 9-Aminoacridine & $90-45-9$ & Dimethyl sulfoxide & Absence & TA1537 & 50 \\
\hline Mitomvcin-C & $50-07-7$ & Distilled water & Absence & TA102 & 0.25 \\
\hline 2-Aminoanthracene & $613-13-8$ & Dimethyl sulfoxide & Presence & $\begin{array}{c}\text { TA1537,TA1535, } \\
\text { TA102,TA100 and } \\
\text { TA98 }\end{array}$ & 20 \\
\hline
\end{tabular}

Key: CAS-Chemical Abstracts Service Registry Number, $\mu g$ - microgram 
Plate incorporation and preincubation methods were conducted according to Maron and Ames ${ }^{19}$ and OECD Guidelines for the Testing of Chemicals no. 471. Following the plate incorporation method, $100 \mu \mathrm{L}$ - Test Item solution at each dose level, vehicle control or reference mutagen solution (positive control), $500 \mu \mathrm{L}-\mathrm{S} 9 \mathrm{mix}$ (for test with metabolic activation) or $0.1 \mathrm{mM}$ Phosphate buffer (for test without metabolic activation) and $100 \mu \mathrm{L}-$ Bacterial suspension were mixed in a test tube and $2 \mathrm{ml}$ overlay agar $\left(47 \pm 2^{\circ} \mathrm{C}\right)$ was added to each tube. The mixture was vortexed and poured on to minimal agar plates. After solidification the plates were incubated in an incubator for 48 hours at $37 \pm 2^{\circ} \mathrm{C}$.

After incubation, plates were observed for background lawn inhibition and counted for revertant colonies. Conditions of the bacterial background lawn was evaluated for evidence of cytotoxicity.

The test substance was considered positive in bacterial reverse mutation assay when there is an increase at least a 2-fold increase in the mean revertants per plate of at least one of these tester strains over the mean revertants per plate of the appropriate vehicle control. This increase in the mean number of revertants per plate must be accompanied by a dose response (minimum of 2 to 3 concentrations) to increasing concentrations of the test item for the tester strainsTA98, TA100 and TA102 whereas for Tester Strains TA1537 and TA1535 there should be at least a 3 -fold increase in the mean revertant per plate of at least one of these tester strains over the mean revertant per plate of the appropriate vehicle control.

A test item for which the results do not meet the above criteria is considered non-mutagenic in this test.

\section{Statistical Analysis}

All the data was expressed in mean \pm SD. In sub-chronic toxicity study, Dunnett's t-test was applied for all heterogeneous data, whereas homogeneous data, which was analysed using ANOVA and showing significance. In recovery groups of main study, t-test was applied for all homogeneous data, whereas heterogeneous data was analysed by Mann Whitney's test. A 95\% confidence level was used to determine statistically significant differences.
In Mammalian Bone Marrow Chromosome Aberration Test, occurrence of structural chromosomal aberrations was evaluated by using one-way analysis of variance. In Mammalian Bone Marrow Micronucleus Test, occurrence of micronuclei in test item treated animals to the results obtained for the vehicle control group was evaluated using one way Analysis of Variance (Dunnett's Method).

\section{RESULTS}

\section{Acute Oral Toxicity Study}

No mortality was observed in the animals of G1 and G2 treated with 300 and $2000 \mathrm{mg} / \mathrm{kg}$ body weight dose throughout the 14 days observation period. The mean body weights of all the animals of G1 and G2 (Step I, Step II, Step III and Step IV) treated with 300 and $2000 \mathrm{mg} / \mathrm{kg}$ body weight respectively, was observed with gain on day 7 and 14, as compared to day 0.No external and internal gross pathological changes were seen in all the animals of G1 (Step I and II) and G2 (Step III and IV) treated with 300 and $2000 \mathrm{mg} / \mathrm{kg}$ body weight respectively, during terminal sacrifice.

\section{Day Repeated Dose Oral Toxicity Study}

No treatment related mortality/morbidity and changes in clinical signs were observed up to $600 \mathrm{mg} / \mathrm{kg}$ body weight group and control in either sex in Dose Range Finding (DRF) study and up to $240 \mathrm{mg} / \mathrm{kg}$ body weight group and control of Main Study.

No treatment related changes were noted in body weight (Table 2), body weight gain percentage and feed consumption up to 600 $\mathrm{mg} / \mathrm{kg}$ body weight in either sex in Dose Range Finding (DRF) study and up to $240 \mathrm{mg} / \mathrm{kg}$ body weight of Main Study. No treatment related changes were noted in functional observational battery/ neurobehavioral observation, foot splay, grip strength and motor activity assessment. There were no statistically significant changes in the neurology, haematological parameters (Table 3), clinical chemistry (Table 4), and the urine parameters.

Visceral examination of the rats of control and other treated groups did not reveal any pathological abnormality except size reduction in testis and epididymideswas observed in one male rat of G4R group. The gross histopathology showed no significant lesions in all dose groups and control group. 
Mutagenic, Genotoxic and Sub Chronic Oral Safety Analysis of Boswellia Serrata Extract (Boswellin ${ }^{\circledR}$ Super)

Table2. Absolute organ weight -90 days sub chronic toxicity study of Boswellia serrata extract

\begin{tabular}{|c|c|c|c|c|c|c|}
\hline Group (N) & G1 (10) & G2 (10) & G3 (10) & G4 (10) & G1R (5) & G4R(5) \\
\hline Dose (mg/Kg) & $\mathbf{0}$ & $\mathbf{6 0}$ & $\mathbf{1 2 0}$ & $\mathbf{2 4 0}$ & $\mathbf{0}$ & $\mathbf{2 4 0}$ \\
\hline Organ (g) & Mean \pm SD & Mean \pm SD & Mean \pm SD & Mean \pm SD & Mean \pm SD & Mean \pm SD \\
\hline Body Weight (g) & $339.5 \pm 32.50$ & $335.5 \pm 29.91$ & $349.9 \pm 30.59$ & $318.7 \pm 43.62$ & $389.2 \pm 33.42$ & $377.4 \pm 43.10$ \\
\hline & $234.6 \pm 22.73$ & $225.3 \pm 29.12$ & $225.0 \pm 16.36$ & $216.0 \pm 15.76$ & $243.0 \pm 15.46$ & 238.2019 .59 \\
\hline Brain & $2.056 \pm 0.106$ & $2.093 \pm 0.117$ & $2.077 \pm 0.080$ & $1.984 \pm 0.091$ & $2.155 \pm 0.092$ & $2.091 \pm 0.121$ \\
\hline & $1.959 \pm 0.091$ & $1.860 \pm 0.122$ & $1.890 \pm 0.035$ & $1.841 \pm 0.129$ & $1.861 \pm 0.080$ & $1.891 \pm 0.067$ \\
\hline Adrenals & $0.056 \pm 0.012$ & $0.050 \pm 0.006$ & $0.056 \pm 0.007$ & $0.054 \pm 0.008$ & $0.051 \pm 0.007$ & $0.047 \pm 0.009$ \\
\hline & $0.064 \pm 0.009$ & $0.067 \pm 0.017$ & $0.074 \pm 0.015$ & $0.071 \pm 0.013$ & $0.059 \pm 0.011$ & $0.064 \pm 0.013$ \\
\hline Testes & $3.158 \pm 0.337$ & $3.215 \pm 0.241$ & $3.390 \pm 0.353$ & $3.064 \pm 0.200$ & $3.256 \pm 0.304$ & $2.821 \pm 1.174$ \\
\hline Epididymides & $1.191 \pm 0.078$ & $1.188 \pm 0.059$ & $1.210 \pm 0.092$ & $1.164 \pm 0.127$ & $1.331 \pm 0.108$ & $1.175 \pm 0.390$ \\
\hline Ovaries with duct & $0.128 \pm 0.013$ & $0.124 \pm 0.019$ & $0.126 \pm 0.012$ & $0.131 \pm 0.017$ & $0.108 \pm 0.012$ & $0.117 \pm 0.011$ \\
\hline Uterus & $0.539 \pm 0.182$ & $0.503 \pm 0.103$ & $0.518 \pm 0.093$ & $0.526 \pm 0.171$ & $0.466 \pm 0.099$ & $0.518 \pm 0.189$ \\
\hline Heart & $1.216 \pm 0.146$ & $1.216 \pm 0.128$ & $1.232 \pm 0.101$ & $1.166 \pm 0.126$ & $1.197 \pm 0.129$ & $1.260 \pm 0.108$ \\
\hline Liver & $0.877 \pm 0.089$ & $0.855 \pm 0.094$ & $0.851 \pm 0.071$ & $0.871 \pm 0.091$ & $0.950 \pm 0.137$ & $0.937 \pm 0.130$ \\
\hline & $10.806 \pm 1.060$ & $10.319 \pm 0.875$ & $11.899 \pm 1.103$ & $10.77 \pm 1.346$ & $12.172 \pm 2.170$ & $12.657 \pm 1.672$ \\
\hline Kidneys & $7.637 \pm 1.052$ & $7.745 \pm 1.274$ & $8.289 \pm 1.073$ & $8.587 \pm 1.421$ & $7.950 \pm 0.721$ & $8.796 \pm 0.876$ \\
\hline & $2.697 \pm 0.279$ & $2.574 \pm 0.268$ & $2.749 \pm 0.194$ & $2.450 \pm 0.280$ & $2.788 \pm 0.308$ & $2.794 \pm 0.129$ \\
\hline Spleen & $1.729 \pm 0.226$ & $1.667 \pm 0.191$ & $1.659 \pm 0.161$ & $1.658 \pm 0.139$ & $1.627 \pm 0.092$ & $1.844 \uparrow * \pm 0.153$ \\
\hline & $0.710 \pm 0.071$ & $0.616 \downarrow * \pm 0.069$ & $0.684 \pm 0.154$ & $0.809 \pm 0.287$ & $0.721 \pm 0.273$ & $0.634 \pm 0.111$ \\
\hline Thymus & $0.531 \pm 0.107$ & $0.559 \pm 0.140$ & $0.585 \pm 0.192$ & $0.651 \pm 0.225$ & $0.495 \pm 0.095$ & $0.499 \pm 0.076$ \\
\hline & $0.286 \pm 0.087$ & $0.270 \pm 0.044$ & $0.233 \pm 0.084$ & $0.233 \pm 0.055$ & $0.249 \pm 0.051$ & $0.241 \pm 0.101$ \\
\hline
\end{tabular}

Keys: $S D=$ Standard deviation, $N=$ Number of animals in group, $\uparrow *=$ Statistically significant increase $(p<$ 0.05), $\downarrow^{*}=$ Statistically significant decrease $(p<0.05)$.

Table3. Hematological parameters at 90 days sub-chronic toxicity study of Boswellia serrata extract

\begin{tabular}{|c|c|c|c|c|c|c|c|}
\hline Group (N) & & G1 (10) & G2 (10) & G3 (10) & G4 (10) & G1R (5) & G4R (5) \\
\hline Dose (mg/kg) & & 0 & 60 & 120 & 240 & 0 & 240 \\
\hline Parameter & Sex & Mean \pm SD & Mean \pm SD & Mean \pm SD & Mean \pm SD & Mean \pm SD & Mean \pm SD \\
\hline \multirow{2}{*}{$\begin{array}{c}\mathrm{RBC} \pm(\mathrm{X} \\
\left.10^{6} / \mu \mathrm{l}\right)\end{array}$} & $\mathrm{M}$ & $8.16 \pm 0.35$ & $8.28 \pm 0.64$ & $8.18 \pm 0.38$ & $8.02 \pm 0.91$ & $9.68 \pm 0.48$ & $7.48 \downarrow^{*} \pm 0.14$ \\
\hline & $\mathrm{F}$ & $8.34 \pm 0.43$ & $8.29 \pm 0.22$ & $8.09 \pm 0.37$ & $7.96 \pm 0.61$ & $8.17 \pm 0.31$ & $7.81 \pm 0.31$ \\
\hline \multirow{2}{*}{ HCT (\%) } & $\mathrm{M}$ & $36.84 \pm 2.25$ & $37.42 \pm 2.99$ & $37.14 \pm 2.04$ & $36.78 \pm 1.78$ & $44.16 \pm 2.47$ & $39.50 \downarrow^{*} \pm 0.80$ \\
\hline & $\mathrm{F}$ & $39.50 \pm 1.99$ & $38.93 \pm 0.73$ & $38.36 \pm 1.92$ & $39.96 \pm 2.21$ & $38.70 \pm 1.60$ & $37.30 \pm 1.49$ \\
\hline \multirow{2}{*}{$\operatorname{MCV}\left(\mu m^{3}\right)$} & $\mathrm{M}$ & $45.11 \pm 1.64$ & $45.21 \pm 1.59$ & $45.41 \pm 2.05$ & $46.41 \pm 6.07$ & $45.64 \pm 3.30$ & $52.86 \uparrow * \pm 1.87$ \\
\hline & $\mathrm{F}$ & $47.38 \pm 0.78$ & $46.98 \pm 0.99$ & $47.42 \pm 1.01$ & $50.35 \pm 3.85$ & $47.32 \pm 1.03$ & $47.76 \pm 0.23$ \\
\hline \multirow{2}{*}{ HGB (g/dl) } & $\mathrm{M}$ & $13.93 \pm 0.82$ & $14.10 \pm 0.93$ & $13.96 \pm 0.66$ & $13.62 \pm 0.71$ & $16.50 \pm 0.74$ & $14.56 \downarrow * \pm 0.43$ \\
\hline & $\mathrm{F}$ & $15.11 \pm 0.60$ & $15.04 \pm 0.29$ & $14.54 \pm 0.77$ & $14.82 \pm 0.82$ & $14.90 \pm 0.46$ & $14.40 \pm 0.37$ \\
\hline \multirow{2}{*}{ MCH (pg) } & $\mathrm{M}$ & $17.06 \pm 0.65$ & $17.05 \pm 0.51$ & $17.09 \pm 0.68$ & $17.14 \pm 1$ & $17.08 \pm 1.06$ & $19.50 \uparrow * \pm 0.81$ \\
\hline & $\mathrm{F}$ & $18.13 \pm 0$. & $18.17 \pm 0.36$ & $17.99 \pm$ & $18.69 \pm$ & $18.24 \pm 0.43$ & $4 \pm 0.40$ \\
\hline \multirow{2}{*}{$\operatorname{MCHC}(\mathrm{g} / \mathrm{dl})$} & $\mathrm{M}$ & 37.8 & 0.72 & .47 & $37.05 \pm 1.12$ & $37.42 \pm 0.78$ & 0.48 \\
\hline & $\mathrm{F}$ & $38.28 \pm 0.56$ & $38.69 \pm 0.54$ & $37.93=$ & $37.17 \downarrow^{*} \pm 1.11$ & $38.54 \pm 0.51$ & \pm 0.80 \\
\hline \multirow{2}{*}{$\begin{array}{c}\text { PLT (X } \\
\left.10^{3} / \mu \mathrm{l}\right) \\
\end{array}$} & $\mathrm{M}$ & $405.40 \pm 71.13$ & $474.00 \pm 90.22$ & $496.50 \pm 105.97$ & $473.90 \pm 120.56$ & $571.20 \pm 58.65$ & $497.20 \downarrow * \pm 33.28$ \\
\hline & $\mathrm{F}$ & $796.00 \pm 170.25$ & $812.10 \pm 120.35$ & $729.70 \pm 87.39$ & $647.80 \downarrow^{*} \pm 147.51$ & $615.20 \pm 73.35$ & $530.40 \pm 49.47$ \\
\hline \multirow{2}{*}{$\begin{array}{c}\text { WBC (X } \\
\left.10^{3} / \mu \mathrm{l}\right)\end{array}$} & $\mathrm{M}$ & $11.50 \pm 2.12$ & $12.38 \pm 2.48$ & $11.05 \pm 3.35$ & $13.61 \pm 4.78$ & $16.08 \pm 4.68$ & 1.62 \\
\hline & $\mathrm{F}$ & 10.65 & $9.60 \pm$ & $10.00 \pm 2.71$ & 10.70 & 1.15 & 1.98 \\
\hline \multirow{2}{*}{$\begin{array}{c}\text { Neutrophil } \\
(\%)\end{array}$} & $\mathrm{M}$ & $13.90 \pm$ & $15.10 \pm 3.28$ & $21.20 \uparrow * \pm 3.68$ & $15.90 \pm 3.70$ & $15.20 \pm 5.76$ & $30 \pm 3.35$ \\
\hline & $\mathrm{F}$ & $16.90 \pm 3.25$ & $15.10 \pm 3.28$ & $16.70 \pm 2.21$ & $14.10 \pm 3.03$ & $18.60 \pm 6.80$ & $17.40 \pm 5.90$ \\
\hline \multirow{2}{*}{$\begin{array}{c}\text { Lymphocyte } \\
(\%)\end{array}$} & $\mathrm{M}$ & $20 \pm 6.20$ & $4.10 \pm 3.45$ & $77.50 \downarrow^{*} \pm 3.84$ & $82.90 \pm 3.67$ & $83.20 \pm 5.63$ & $78.20 \pm 4.02$ \\
\hline & $\mathrm{F}$ & .40 & 3.45 & $81.80 \pm 2.82$ & $85.40 \uparrow * \pm 3.20$ & 7.73 & \pm 6.14 \\
\hline \multirow{2}{*}{$\begin{array}{c}\text { Monocyte } \\
(\%)\end{array}$} & $\mathrm{M}$ & $0.30 \pm 0.48$ & $0.60 \pm 0.20$ & & $0.40 \pm 0.52$ & $0.80 \pm 0.84$ & \pm 0.55 \\
\hline & $\mathrm{F}$ & $0.90 \pm 0.74$ & $0.20 \downarrow * \pm 0.42$ & $0.70 \pm 0.48$ & $0.30 \downarrow^{*} \pm 0.48$ & $0.20 \pm 0.45$ & $0.40 \pm 0.55$ \\
\hline \multirow{2}{*}{$\begin{array}{c}\text { Eosinophil } \\
(\%)\end{array}$} & $\mathrm{M}$ & $0.60 \pm 0.52$ & $0.80 \pm 0.79$ & $0.60 \pm 0.70$ & $0.80 \pm 0.63$ & $0.80 \pm 1.30$ & $1.40 \pm 1.14$ \\
\hline & $\mathrm{F}$ & $0.90 \pm 0.57$ & $0.60 \pm 0.52$ & $0.80 \pm 0.63$ & $0.20 \downarrow * \pm 0.42$ & $0.80 \pm 0.84$ & $1.00 \pm 0.71$ \\
\hline \multirow{2}{*}{ Basophil (\%) } & $\mathrm{M}$ & $0.00 \pm 0.00$ & $0.00 \pm 0.00$ & & $0.00 \pm 0.00$ & $0.00 \pm 0.00$ & $0.00 \pm 0.00$ \\
\hline & $\mathrm{F}$ & $0.00 \pm 0.00$ & $0.00 \pm 0.00$ & & $0.00 \pm 0.00$ & $0.00 \pm 0.00$ & $0.00 \pm 0.00$ \\
\hline \multirow{2}{*}{ PT(sec.) } & $\mathrm{M}$ & $22.60 \pm 2.96$ & $28.10 \pm 24.96$ & $22.19 \pm 4.54$ & $21.58 \pm 7.02$ & $18.02 \pm 0.40$ & $19.42 \pm 2.01$ \\
\hline & $\mathrm{F}$ & $23.69 \pm 7.12$ & $20.83 \pm 1.99$ & $20.51 \pm 3.69$ & $21.05 \pm 3.81$ & $21.25 \pm 0.63$ & $20.61 \pm 1.54$ \\
\hline \multirow{2}{*}{ aPTT(sec.) } & $\mathrm{M}$ & $30.75 \pm 3.00$ & $22.70 \downarrow^{*} \pm 3.48$ & $22.51 \downarrow * \pm 4.61$ & $27.42 \pm 5.04$ & $22.25 \pm 4.07$ & $23.01 \pm 4.88$ \\
\hline & $\mathrm{F}$ & $22.01 \pm 4.61$ & $21.42 \pm 1.85$ & $21.40 \pm 1.66$ & $21.04 \pm 3.42$ & $19.37 \pm 2.50$ & $18.16 \pm 3.31$ \\
\hline
\end{tabular}

Keys: $S D=$ Standard deviation, $N=$ Number of animals in group, $\uparrow *=$ Statistically significant increase $(p<$ $0.05), \downarrow^{*}=$ Statistically significant decrease $(p<0.05)$. 
Mutagenic, Genotoxic and Sub Chronic Oral Safety Analysis of Boswellia Serrata Extract (Boswellin ${ }^{\circledR}$ Super)

Table4. Clinical Biochemistry parameters at 90 days sub-chronic toxicity study

\begin{tabular}{|c|c|c|c|c|c|c|c|}
\hline Group (N) & & G1 (10) & G2 (10) & G3 (10) & G4 (10) & G1R (5) & G4R (5) \\
\hline Dose (mg/kg) & & o & 60 & 120 & 240 & 0 & 240 \\
\hline Parameter & Sex & Mean \pm SD & Mean \pm SD & Mean \pm SD & Mean \pm SD & Mean \pm SD & Mean \pm SD \\
\hline \multirow{2}{*}{$\operatorname{ALP}(\mathbf{U} / \mathbf{I})$} & $\mathrm{M}$ & $214.49 \pm 70.74$ & $212.07 \pm 40.57$ & $202.27 \pm 60.14$ & $193.15 \pm 70.78$ & $140.84 \pm 29.96$ & $138.72 \pm 29.62$ \\
\hline & $\mathrm{F}$ & $96.73 \pm 25.74$ & $106.09 \pm 22.35$ & $149.29 \pm 94.81$ & $144.62 \uparrow * \pm 29.37$ & $91.14 \pm 36.58$ & $82.22 \pm 11.61$ \\
\hline \multirow{2}{*}{$\operatorname{ALT}(\mathbf{U} / \mathbf{I})$} & $\mathrm{M}$ & $61.30 \pm 13.55$ & $60.26 \pm 6.46$ & $52.91 \pm 4.61$ & $64.19 \pm 10.29$ & $59.48 \pm 3.64$ & $46.08 \downarrow * \pm 3.48$ \\
\hline & $\mathrm{F}$ & $50.56 \pm 6.85$ & $48.19 \pm 5.96$ & $52.26 \pm 10.60$ & $50.75 \pm 10.15$ & $51.88 \pm 6.95$ & $45.80 \pm 6.80$ \\
\hline \multirow{2}{*}{$\operatorname{Albumin}(\mathrm{g} / \mathrm{dl})$} & $\mathrm{M}$ & $3.15 \pm 0.21$ & $3.19 \pm 0.21$ & $3.17 \pm 0.21$ & $3.10 \pm 0.18$ & $3.24 \pm 0.14$ & $3.28 \pm 0.15$ \\
\hline & $\mathrm{F}$ & $3.34 \pm 0.24$ & $3.32 \pm 0.16$ & $3.22 \pm 0.36$ & $3.26 \pm 0.18$ & $3.70 \pm 0.22$ & $3.54 \pm 0.20$ \\
\hline \multirow{2}{*}{$\operatorname{AST}(\mathbf{U} / \mathbf{I})$} & $\mathrm{M}$ & $152.26 \pm 22.95$ & $167.85 \pm 18.68$ & $150.01 \pm 22.82$ & $179.07 \pm 43.14$ & $108.40 \pm 8.24$ & $103.92 \pm 20.04$ \\
\hline & $\mathrm{F}$ & $159.90 \pm 42.15$ & $128.89 \pm 16.81$ & $148.85 \pm 27.88$ & $145.63 \pm 25.64$ & $203.64 \pm 42.79$ & $181.88 \pm 38.25$ \\
\hline \multirow{2}{*}{ Bileacids $(\mu \mathrm{mol} / \mathrm{L})$} & $\mathrm{M}$ & $48.55 \pm 46.94$ & $34.10 \pm 14.20$ & $31.06 \pm 14.93$ & $38.75 \pm 21.93$ & $5.96 \pm 2.03$ & $8.08 \pm 4.11$ \\
\hline & $\mathrm{F}$ & $20.02 \pm 13.62$ & $17.64 \pm 15.13$ & $13.92 \pm 7.47$ & $17.36 \pm 15.35$ & $10.44 \pm 5.78$ & $7.30 \pm 2.81$ \\
\hline \multirow{2}{*}{ Urea(mg/dl) } & $\mathrm{M}$ & $52.12 \pm 13.52$ & $51.51 \pm 6.88$ & $53.16 \pm 12.88$ & $60.52 \pm 9.32$ & $32.00 \pm 2.28$ & $32.46 \pm 2.54$ \\
\hline & $\mathrm{F}$ & $45.31 \pm 8.33$ & $44.13 \pm 4.94$ & $48.12 \pm 7.03$ & $53.69 \pm 12.43$ & $42.60 \pm 6.82$ & $43.22 \pm 2.37$ \\
\hline \multirow{2}{*}{ Calcium(mg/dl) } & $\mathrm{M}$ & $9.72 \pm$ & & $9.74 \pm$ & $9.50 \pm 0.36$ & $9.71 \pm 0.14$ & $9.71 \pm 0.28$ \\
\hline & $\mathrm{F}$ & $9.94 \pm 0$ & $9.85 \pm$ & $9.83 \pm 0$ & $9.68 \pm 0$ & $10.34 \pm 0.31$ & $10.12 \pm 0.26$ \\
\hline \multirow{2}{*}{ Cholesterol(mg/dl) } & $\mathrm{M}$ & $45.47 \pm 11.75$ & $53.26 \pm 10.34$ & $56.02 \pm 9.59$ & $53.32 \pm 8.49$ & $52.16 \pm 6.56$ & $60.66 \pm 16.88$ \\
\hline & $\mathrm{F}$ & $47.96 \pm 7.26$ & $54.46 \pm 8.37$ & $47.45 \pm 12.63$ & $54.27 \pm 11.33$ & $56.78 \pm 9.85$ & $54.36 \pm 11.25$ \\
\hline \multirow{2}{*}{ Creatinine(mg/dl) } & $\mathrm{M}$ & $0.58 \pm 0.04$ & $0.60 \pm 0.04$ & $0.58 \pm 0.04$ & $0.60 \pm 0.05$ & $0.65 \pm 0.03$ & $0.59 \downarrow * \pm 0.01$ \\
\hline & $\mathrm{F}$ & $0.58 \pm 0$ & $0.58 \pm$ & $0.60 \pm($ & $0.57 \pm 0$ & $0.51 \pm 0.04$ & $0.47 \pm 0.02$ \\
\hline \multirow{2}{*}{ Glucose(mg/dl) } & $\mathrm{M}$ & $59.09 \pm 9.16$ & $68.82 \pm 7.33$ & $62.04 \pm 7.40$ & $63.23 \pm 7$ & $107.80 \pm 18.44$ & $90.26 \pm 8.43$ \\
\hline & $\mathrm{F}$ & $76.54 \pm 14.81$ & $69.03 \pm 8$ & $66.78 \pm 7.55$ & $69.79 \pm 1$ & $71.94 \pm 21.57$ & $62.42 \pm 6.46$ \\
\hline \multirow{2}{*}{$\begin{array}{c}\text { Totalbilirubin(mg } \\
\text { /dl) }\end{array}$} & $\mathrm{M}$ & & & $0.13 \pm$ & $0.12 \pm 0$ & $0.12 \pm 0.01$ & $0.12 \pm 0.02$ \\
\hline & $\mathrm{F}$ & $0.13 \pm 0$ & & $0.10 \pm($ & 0.1 & $0.15 \pm 0.03$ & $0.16 \pm 0.06$ \\
\hline \multirow{2}{*}{ Totalprotein(g/dl) } & $\mathrm{M}$ & $7.09 \pm 0$ & $7.56 \uparrow * \pm 0.31$ & $7.38 \pm$ & $7.65 \uparrow * \pm 0$ & $7.44 \pm 0.12$ & $7.25 \pm 0.25$ \\
\hline & $\mathrm{F}$ & $7.48 \pm 0$ & $7.46 \pm 0.30$ & $7.38 \pm 0$. & $7.76 \pm 0.43$ & $7.71 \pm 0.36$ & $7.64 \pm 0.34$ \\
\hline \multirow{2}{*}{$\begin{array}{l}\text { Triglyceride(mg/ } \\
\text { dl) }\end{array}$} & $\mathrm{M}$ & $58.72 \pm 10.92$ & & $62.72 \pm 1$ & & $57.04 \pm 15.33$ & $60.02 \pm 12.59$ \\
\hline & $\mathrm{F}$ & $54.69 \pm 10.67$ & $61.92 \pm 18.05$ & $49.43 \pm 12.28$ & $50.20 \pm 13.84$ & $54.20 \pm 5.58$ & $56.04 \pm 8.62$ \\
\hline \multirow{2}{*}{$\begin{array}{l}\text { Phosphorus(mg/ } \\
\text { dl) }\end{array}$} & $\mathrm{M}$ & & & & & 0.55 & $5.58 \pm 0.79$ \\
\hline & $\mathrm{F}$ & $5.03 \pm$ & 4.62 & $5.54 \pm 0$ & $5.55 \pm 0$ & $5.07 \pm 0.49$ & .00 \\
\hline \multirow{2}{*}{$\mathbf{L D}(\mathbf{U} / \mathbf{I})$} & M & 2681.30 & $2626.00 \pm$ & $2607.90 \pm 648.65$ & $2961.40 \pm 13$ & $1037.40 \pm 200.53$ & $1091.80 \pm 322.80$ \\
\hline & $\mathrm{F}$ & 2602.5 & 1758.60 & $1974.10 \pm$ & $1972.60 \pm$ & $2597.6 \pm 967.9$ & 3164.60 \\
\hline \multirow{2}{*}{ GGT(U/I) } & $\mathrm{M}$ & & & $2.60 \pm$ & $2.62 \pm 0.47$ & $2.23 \pm 0.58$ & $2.05 \pm 0.74$ \\
\hline & $\mathrm{F}$ & $2.42 \pm 0.34$ & $2.55 \pm 0.32$ & $4.11 \pm 4.18$ & $3.29 \uparrow * \pm 0.34$ & $1.93 \pm 0.28$ & $2.33 \pm 0.36$ \\
\hline \multirow{2}{*}{ CK(U/I) } & $\mathrm{M}$ & $567.70 \pm 124.54$ & $598.10 \pm 347.72$ & $519.10 \pm 170.59$ & $475.70 \pm 166.29$ & $244.20 \pm 53.31$ & $225.60 \pm 65.51$ \\
\hline & $\mathrm{F}$ & $621.80 \pm 287.10$ & $379.10 \pm 120.75$ & $391.70 \pm 172.32$ & $289.10 \downarrow * \pm 100.40$ & $512.0 \pm 207.7$ & $462.80 \pm 83.16$ \\
\hline \multirow{2}{*}{$\mathrm{Na}(\mathrm{mmol} / \mathrm{l})$} & $\mathrm{M}$ & & & $150.50 \downarrow * \pm 1.90$ & $151.90 \pm 0.74$ & $143.40 \pm 0.89$ & $144.60 \pm 1.52$ \\
\hline & $\mathrm{F}$ & $141.90 \pm 1.10$ & & $146.60 \uparrow * \pm 1.58$ & $147.80 \uparrow * \pm 1.55$ & $145.0 \pm 1.0$ & $145.00 \pm 2.55$ \\
\hline \multirow{2}{*}{$\mathbf{K}(\mathbf{m m o l} / \mathbf{l})$} & $\mathrm{M}$ & & $5.79 \pm 0.45$ & $5.73 \pm 0.46$ & $5.64 \pm 0.31$ & $4.80 \pm 0.16$ & $5.22 \uparrow * \pm 0.26$ \\
\hline & $\mathrm{F}$ & $5.16 \pm 0.55$ & $5.10 \pm 0.34$ & $5.15 \pm 0.21$ & $5.06 \pm 0.69$ & $4.88 \pm 0.16$ & $4.94 \pm 0.52$ \\
\hline \multirow{2}{*}{$\mathrm{Cl}(\mathrm{mmol} / \mathrm{l})$} & $\mathrm{M}$ & $111.30 \pm 2.00$ & $109.90 \pm 1.66$ & $108.20 \downarrow * \pm 1.55$ & $110.10 \pm 1.10$ & $104.60 \pm 1.14$ & $105.60 \pm 1.52$ \\
\hline & $\mathrm{F}$ & $103.70 \pm 1.42$ & $103.10 \pm 1.10$ & $107.80 \uparrow * \pm 1.81$ & $108.00 \uparrow * \pm 2.16$ & $106.6 \pm 0.89$ & $106.20 \pm 2.59$ \\
\hline \multirow{2}{*}{ BUN(mg/dl) } & $\mathrm{M}$ & $24.36 \pm 6.32$ & $24.07 \pm 3.22$ & $24.84 \pm 6.02$ & $28.28 \pm 4.35$ & $14.95 \pm 1.06$ & $15.17 \pm 1.19$ \\
\hline & $\mathrm{F}$ & $21.17 \pm 3.89$ & $20.62 \pm 2.31$ & $22.49 \pm 3.28$ & $25.09 \pm 5.81$ & $19.91 \pm 3.19$ & $20.20 \pm 1.11$ \\
\hline & $\mathrm{M}$ & $3.95 \pm 0.38$ & $4.37 \pm 0.36$ & $4.21 \pm 0.44$ & $4.55 \uparrow * \pm 0.28$ & $4.19 \pm 0.21$ & $3.96 \pm 0.14$ \\
\hline & $\mathrm{F}$ & $4.14 \pm 0.36$ & $4.14 \pm 0.27$ & $4.16 \pm 0.29$ & $4.50 \pm 0.48$ & $4.02 \pm 0.22$ & $4.10 \pm 0.25$ \\
\hline & $\mathrm{M}$ & & $0.74 \pm 0.09$ & $0.76 \pm 0.11$ & $0.68 \pm 0.07$ & $0.77 \pm 0.07$ & $0.83 \pm 0.03$ \\
\hline & $\mathrm{F}$ & $0.81 \pm 0.08$ & $0.80 \pm 0.07$ & $0.78 \pm 0.08$ & $0.74 \pm 0.09$ & $0.92 \pm 0.06$ & 0.860 .07 \\
\hline
\end{tabular}

Keys: $S D=$ Standard deviation, $N=$ Number of animals in group, $\uparrow *=$ Statistically significant increase $(p<$ $0.05), \downarrow^{*}=$ Statistically significant decrease $(p<0.05)$.

Mammalian Bone Marrow Chromosome Aberration Test of Boswellia serrata Extract

In dose range finding study and in main study, animals (male and female) treated with 500, 1000 and $2000 \mathrm{mg}$ of Boswellia serrata Extract $/ \mathrm{kg}$ body weight, positive control $(50 \mathrm{mg} / \mathrm{kg}$ body weight cyclophosphamide monohydrate) and $0.5 \% \quad(\mathrm{w} / \mathrm{v})$ Carboxymethyl Cellulose (vehicle control) appeared normal, no mortality observed, throughout experimental period.

The body weight of the animals was within the range of commonly recorded for this strain and age. Mean and Standard deviation for individual animal body weight on the day of dosing were within the range.

No bone marrow toxicity i.e. no significant decrease in the mitotic index was observed in treatment groups (treated at the dose levels of 500, 1000 and $2000 \mathrm{mg}$ of Boswellia serrata Extract / kg body weight) when compared to concurrent vehicle control group (Table 5 and 6).

No significant increase in number of structural chromosomal aberrations was observed in either male or female animals treated at the dose levels 
of 500, 1000 and $2000 \mathrm{mg}$ of Boswellia serrata Extract $/ \mathrm{kg}$ body weight when compared to concurrent vehicle control group(Table 7).

Significant increase in number of structural chromosomal aberrations was observed in positive control group, treated with $50 \mathrm{mg}$ of Cyclophosphamide monohydrate / kg body weight when compared to concurrent vehicle control group, which represent the sensitivity of test system to positive control (Table 7).

Table5. Mitotic index after Boswellia administration-Dose Range Finding Study

\begin{tabular}{|c|c|c|c|c|}
\hline \multirow[t]{2}{*}{ Group } & \multirow{2}{*}{$\begin{array}{c}\text { Dose Level } \\
\text { (mg/kg B.wt) }\end{array}$} & \multirow[t]{2}{*}{ Sex } & \multicolumn{2}{|c|}{ Mitotic Index } \\
\hline & & & Mean & SD \\
\hline \multirow{2}{*}{ G1 } & \multirow{2}{*}{ 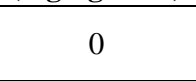 } & $\mathrm{M}$ & 5.40 & 0.36 \\
\hline & & $\mathrm{F}$ & 5.10 & 0.30 \\
\hline \multirow{2}{*}{ G2 } & \multirow{2}{*}{500} & $\mathrm{M}$ & 5.63 & 0.67 \\
\hline & & $\mathrm{F}$ & 5.00 & 0.30 \\
\hline \multirow{2}{*}{ G3 } & \multirow{2}{*}{1000} & $\mathrm{M}$ & 5.37 & 0.31 \\
\hline & & $\mathrm{F}$ & 4.97 & 0.21 \\
\hline \multirow{2}{*}{ G4 } & \multirow{2}{*}{2000} & $\mathrm{M}$ & 5.67 & 0.42 \\
\hline & & $\mathrm{F}$ & 5.03 & 0.31 \\
\hline
\end{tabular}

Key: $\mathrm{mg}=$ milligram, $\mathrm{kg}=$ kilogram, $\mathrm{b} . \mathrm{wt}=$ Body weight $S D=$ Standard Deviation

Table6. Mitotic index after Boswellia administration-Main Study

\begin{tabular}{|c|c|c|c|c|}
\hline \multirow[t]{2}{*}{ Group } & \multirow{2}{*}{$\begin{array}{c}\text { Dose Level } \\
\text { (mg/kg B.wt) }\end{array}$} & \multirow[t]{2}{*}{ Sex } & \multicolumn{2}{|c|}{ Mitotic Index } \\
\hline & & & Mean & SD \\
\hline \multirow{2}{*}{ G1 } & \multirow{2}{*}{0} & $\mathrm{M}$ & 5.11 & 0.45 \\
\hline & & $\mathrm{F}$ & 4.62 & 0.28 \\
\hline \multirow{2}{*}{$\mathrm{G} 2$} & \multirow{2}{*}{500} & $\mathrm{M}$ & 4.76 & 0.33 \\
\hline & & $\mathrm{F}$ & 4.91 & 0.45 \\
\hline \multirow{2}{*}{ G3 } & \multirow{2}{*}{1000} & $\mathrm{M}$ & 5.17 & 0.41 \\
\hline & & $\mathrm{F}$ & 4.80 & 0.37 \\
\hline \multirow{2}{*}{ G4 } & \multirow{2}{*}{2000} & $\mathrm{M}$ & 5.04 & 0.39 \\
\hline & & $\mathrm{F}$ & 4.83 & 0.27 \\
\hline \multirow{2}{*}{$\begin{array}{l}\text { G5 } \\
\text { (PC) }\end{array}$} & \multirow{2}{*}{$50^{\#}$} & $\mathrm{M}$ & 4.69 & 0.26 \\
\hline & & $\mathrm{F}$ & 4.94 & 0.45 \\
\hline
\end{tabular}

Key: $m g=$ milligram, $\mathrm{kg}=$ kilogram, $b ., w t=$ Body weight $\mathrm{SD}=$ Standard Deviation,

${ }^{\#}=$ Cyclophosphamide monohydrate, $P C=$ Positive control

Table7. Summary Data on Chromosome Aberrations Main Study

\begin{tabular}{|c|c|c|c|c|c|}
\hline \multirow{3}{*}{$\begin{array}{l}\text { Group } \\
\text { No. }\end{array}$} & \multirow{3}{*}{$\begin{array}{c}\text { Dose Level } \\
\text { (mg/kg B.wt) }\end{array}$} & \multirow{3}{*}{ Sex } & \multicolumn{2}{|c|}{ \% Structural Aberrations } & \multirow{2}{*}{$\begin{array}{c}\text { Numerical } \\
\text { Aberrations }\end{array}$} \\
\hline & & & Including Gaps & Excluding Gaps & \\
\hline & & & Mean \pm SD & Mean \pm SD & Mean \pm SD \\
\hline \multirow{2}{*}{$\begin{array}{c}\text { G1 } \\
\text { (VC) }\end{array}$} & \multirow{2}{*}{0} & $\mathrm{M}$ & $0.00 \pm 0.00$ & $0.00 \pm 0.00$ & $0.00 \pm 0.00$ \\
\hline & & $\mathrm{F}$ & $0.00 \pm 0.00$ & $0.00 \pm 0.00$ & $0.00 \pm 0.00$ \\
\hline \multirow[t]{2}{*}{ G2 } & \multirow[t]{2}{*}{500} & $\mathrm{M}$ & $0.00 \pm 0.00$ & $0.00 \pm 0.00$ & $0.00 \pm 0.00$ \\
\hline & & $\mathrm{F}$ & $0.00 \pm 0.00$ & $0.00 \pm 0.00$ & $0.00 \pm 0.00$ \\
\hline \multirow[t]{2}{*}{ G3 } & \multirow[t]{2}{*}{1000} & $\mathrm{M}$ & $0.00 \pm 0.00$ & $0.00 \pm 0.00$ & $0.00 \pm 0.00$ \\
\hline & & $\mathrm{F}$ & $0.00 \pm 0.00$ & $0.00 \pm 0.00$ & $0.00 \pm 0.00$ \\
\hline \multirow[t]{2}{*}{ G4 } & \multirow[t]{2}{*}{2000} & $\mathrm{M}$ & $0.20 \pm 0.45$ & $0.20 \pm 0.45$ & $0.00 \pm 0.00$ \\
\hline & & $\mathrm{F}$ & $0.00 \pm 0.00$ & $0.00 \pm 0.00$ & $0.00 \pm 0.00$ \\
\hline \multirow{2}{*}{$\begin{array}{l}\text { G5 } \\
\text { (PC) }\end{array}$} & \multirow[t]{2}{*}{$50^{\#}$} & $\mathrm{M}$ & $7.20 \pm 0.45$ & $6.80 \pm 0.84$ & $0.00 \pm 0.00$ \\
\hline & & $\mathrm{F}$ & $7.60 \pm 1.14$ & $6.60 \pm 0.55$ & $0.00 \pm 0.00$ \\
\hline
\end{tabular}

Key: $m g=$ milligram, $k g=$ kilogram, $b . w t=$ Body weight, $M=$ Male, $F=$ Female, $S D=$ Standard Deviation, ${ }^{\#}=$ Cyclophosphamide monohydrate, $V C=$ Vehicle control, $P C=$ Positive control

Mammalian Bone Marrow Micronucleus Test of Boswellia serrata Extract in Mice

All the animals of vehicle control, treatment (treated at the dose levels of 500, 1000 and 2000 mg of Boswellia serrata Extract $/ \mathrm{kg}$ body weight) and positive control groups were observed to be normal throughout experimental period.

No toxicity to bone marrow (i.e. significant decrease in the $\mathrm{P} / \mathrm{E}$ ratio) was observed in any of the treatment groups (treated at the dose levels of 500, 1000 and $2000 \mathrm{mg}$ of Boswellia serrata Extract $\mathrm{mg} / \mathrm{kg}$ body weight), when compared to concurrent vehicle control group.

No statistically significant increase in number of micronucleated polychromatic erythrocytes (\% MNPCE) was observed in either male or female animals treated at the dose levels 500, 1000 and $2000 \mathrm{mg}$ of Boswellia serrata Extract $\mathrm{mg} / \mathrm{kg}$ body weight when compared to concurrent vehicle control group in both DRF study and main study (Table 8 and 9). 
Mutagenic, Genotoxic and Sub Chronic Oral Safety Analysis of Boswellia Serrata Extract (Boswellin ${ }^{\circledR}$ Super)

Statistically significant increase in number of micronucleus (\% MNPCE) was observed in Cyclophosphamide monohydrate $/ \mathrm{kg}$ body positive control group (treated with $50 \mathrm{mg}$ of weight) in main study (Table 9).

Table8. Proportion of Immature Erythrocytes among Total Erythrocytes (dose range finding study)

\begin{tabular}{|c|c|c|c|}
\hline Group & Dose (mg/kg b. wt) & Sex & Mean P/E \\
\hline \multirow[t]{2}{*}{ G1 } & \multirow[t]{2}{*}{$\mathrm{O}(\mathrm{VC})$} & $\mathbf{M}$ & 0.568 \\
\hline & & $\mathbf{F}$ & 0.601 \\
\hline \multirow[t]{2}{*}{ G2 } & \multirow[t]{2}{*}{500} & $\mathbf{M}$ & 0.552 \\
\hline & & $\mathbf{F}$ & 0.538 \\
\hline \multirow[t]{2}{*}{ G3 } & \multirow[t]{2}{*}{1000} & $\mathbf{M}$ & 0.579 \\
\hline & & $\mathbf{F}$ & 0.544 \\
\hline \multirow[t]{2}{*}{ G4 } & \multirow[t]{2}{*}{2000} & M & 0.542 \\
\hline & & $\mathbf{F}$ & 0.542 \\
\hline
\end{tabular}

Key: $m g$ =milligram, $\mathrm{kg}=$ kilogram, $\mathrm{b} . w t=$ body weight,$M=$ Male, $F=$ Female, PCE $=$ Polychromatic Erythrocytes, $E=$ Total Number of Erythrocytes, $P / E=$ Ratio of Polychromatic Erythrocytes to Total Number of Erythrocytes.

Table9. P/E Ratio and Mean and SD of \% Micronucleated Polychromatic Erythrocytes (MNPCE) (Main Study)

\begin{tabular}{|c|c|c|c|c|c|c|c|}
\hline \multirow{2}{*}{$\begin{array}{l}\text { Group } \\
\text { No. }\end{array}$} & \multirow{2}{*}{$\begin{array}{l}\text { Dose (mg/kg } \\
\text { b.wt) }\end{array}$} & \multirow[t]{2}{*}{ Sex } & \multirow[t]{2}{*}{$\mathbf{P} / \mathbf{E}$} & \multirow[t]{2}{*}{ \% MNPCE } & \multirow{2}{*}{$\begin{array}{c}\text { No. of } \\
\text { MNPCE/Group }\end{array}$} & \multicolumn{2}{|c|}{ MNPCE } \\
\hline & & & & & & Mean & SD \\
\hline \multirow{2}{*}{ G1 } & \multirow[b]{2}{*}{$0(\mathrm{VC})$} & $\mathbf{M}$ & 0.5516 & 0.00 & 0 & 0.00 & 0.00 \\
\hline & & $\mathbf{F}$ & 0.5414 & 0.00 & 0 & 0.00 & 0.00 \\
\hline \multirow{2}{*}{ G2 } & \multirow{2}{*}{500} & M & 0.5336 & 0.00 & 0 & 0.00 & 0.00 \\
\hline & & $\mathbf{F}$ & 0.5208 & 0.00 & 0 & 0.00 & 0.00 \\
\hline \multirow{2}{*}{ G3 } & \multirow{2}{*}{1000} & $\mathbf{M}$ & 0.5274 & 0.19 & 1 & 0.20 & 0.45 \\
\hline & & $\mathbf{F}$ & 0.5244 & 0.00 & 0 & 0.00 & 0.00 \\
\hline \multirow{2}{*}{ G4 } & \multirow{2}{*}{2000} & $\mathbf{M}$ & 0.5266 & 0 & 0 & 0.00 & 0.00 \\
\hline & & $\mathbf{F}$ & 0.531 & 0.174 & 1 & 0.20 & 0.45 \\
\hline \multirow{2}{*}{ G5 } & \multirow{2}{*}{$50(\mathrm{PC})$} & $\mathbf{M}$ & 0.5198 & 11.638 & 62 & 12.40 & 1.14 \\
\hline & & $\mathbf{F}$ & 0.535 & 11.4 & 57 & 11.40 & 1.82 \\
\hline
\end{tabular}

Key: $\mathrm{mg}=$ milligram, $\mathrm{kg}=$ kilogram, $\mathrm{b} . \mathrm{wt}=$ body weight, $P C E=$ Polychromatic Erythrocytes, NCE = Normochromatic Erythrocytes, P/E = Ratio of Polychromatic Erythrocytes to Total Number of Erythrocytes, MNPCE = Micronucleated Polychromatic Erythrocytes, $P C=$ positive control $($ Cyclophosphamide monohydrate), $V C=$ Vehicle control, $S D=$ Standard Deviation.

\section{Bacterial Reverse Mutation Test of Boswellia} serrata Extract

Trial I: In all the tester strains, there was no increase in the number of revertant colonies and no background lawn reduction up to the highest tested concentration i.e $5000 \mu \mathrm{g} / \mathrm{plate}$, when compared to vehicle control (Figure 1).

Trial II: The spontaneous revertant colonies were within the acceptable range and did not show any increase in revertant colony count with respect to dose concentration in all the tester strains. In the all the tester strains, slight precipitation was observed at highest tested in the presence of metabolic activation system (Figure 2 and 3).

There was no reduction in background lawn in the all the tested concentrations up to 5000 $\mu \mathrm{g} /$ plate in the presence of metabolic activation when compared to vehicle control plates.

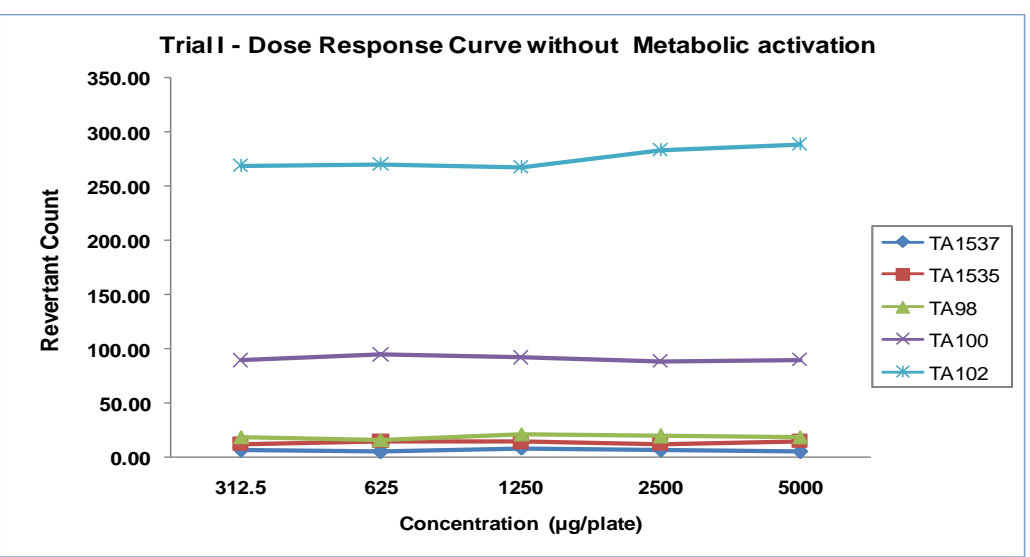

Figure1. Bacterial Reverse Mutation Test of Boswellia serrata Extract; Dose response curve Trial I 


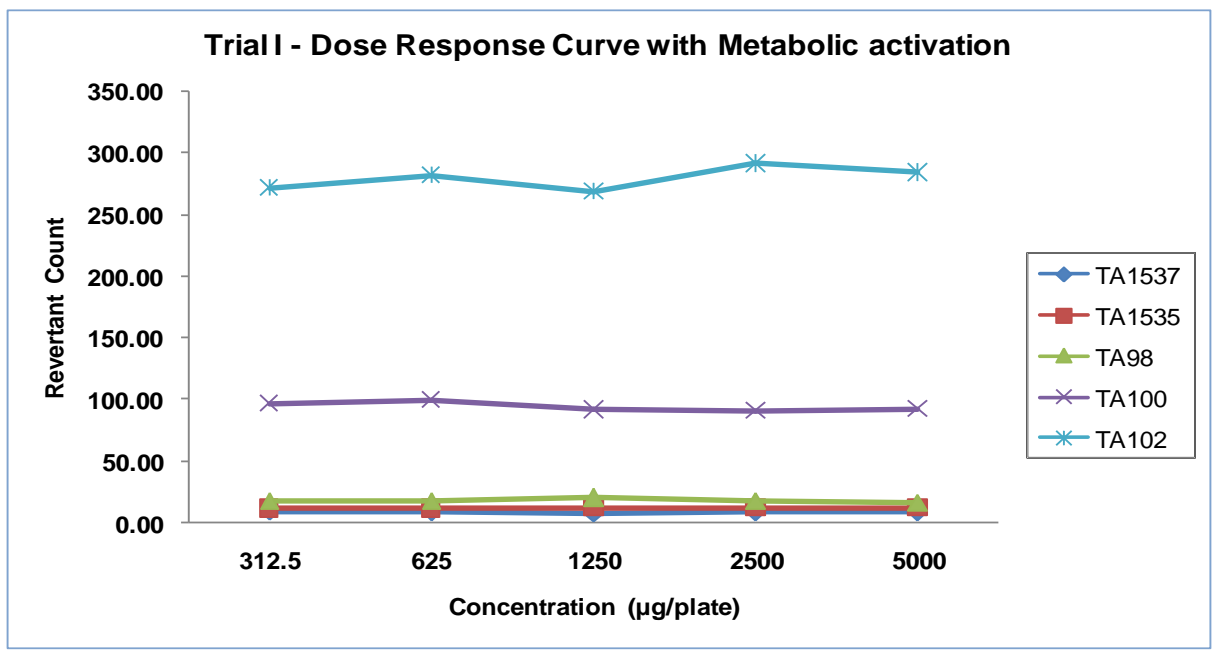

Figure2. Bacterial Reverse Mutation Test of Boswellia serrata Extract; Dose Response Curve Trial II

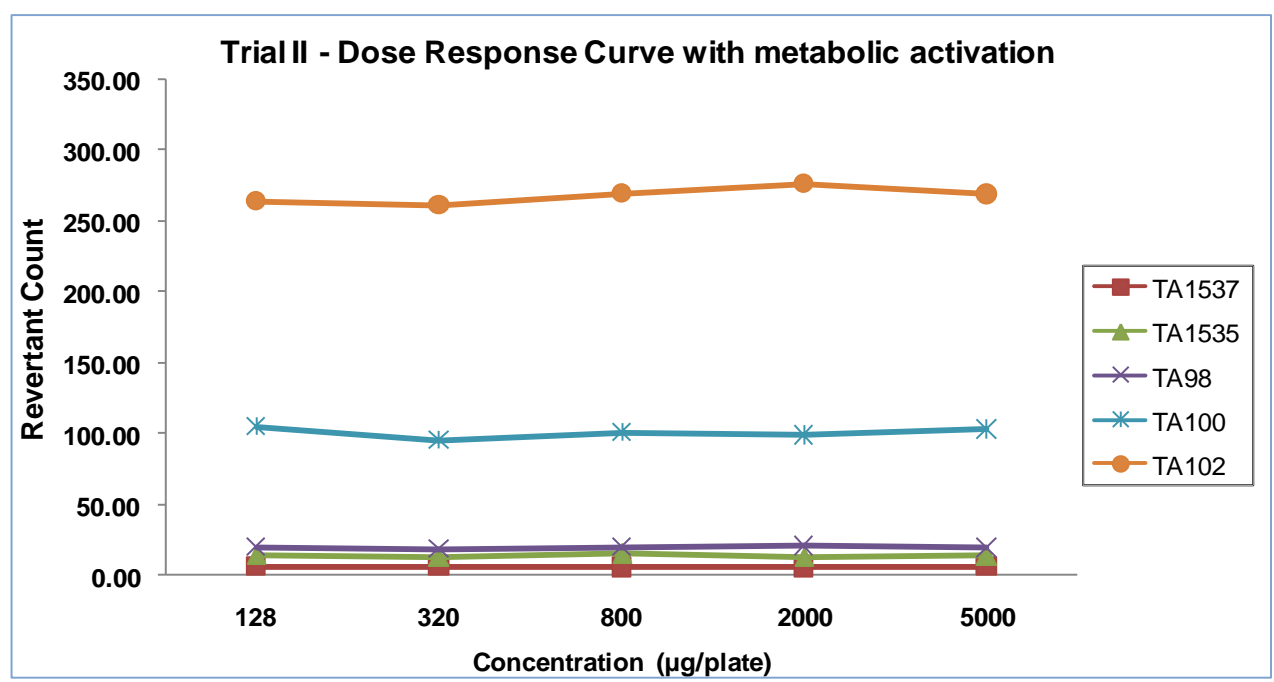

Figure3. Bacterial Reverse Mutation Test of Boswellia serrata Extract; Dose response curve trial II - with metabolic activation

\section{DISCUSSION}

Boswellia serrata a traditional medicinal herb is known to have Boswellic acids as major constituents in its gum extract ${ }^{20}$.A lot of preclinical and clinical studies has been conducted to evaluate its efficacy in the indications like osteoarthritis, crohn's disease, autoimmuneencephalitis and many other inflammatory conditions ${ }^{11}$. The present study aimed to study the mutagenicity of Boswellia serrata extract in terms of bacterial reverse mutation test, bacterial chromosome aberration test, micronucleus test, and in vivo safety by acute and sub chronic toxicity study.

As bone marrow is a highly vascularized tissue and can be readily isolated and processed due to rapidly cycling cells, it has been used as the standard to test the mutagenic hazards in various animal model ${ }^{21}$. In this study, all the animals were found to be normal, and no bone marrow toxicity was observed with the Boswellia serrata extract. Also, number of structural chromosomal aberrations didn't increase significantly inall animals treated with Boswellia serrata Extract when compared to concurrent vehicle control group and positive control group.

The Ames test detects the point mutations including substitution, addition or deletion of one or multiple DNA base pair using amino acid-requiring strains of $S$. typhimurium and $E$. coli $^{22}$.In this study, the tester strains TA98, TA100, TA1537, TA1535 and TA102 were treated with test item, in the presence $(5 \%$ and $10 \% \mathrm{v} / \mathrm{v}$ S9 Mix) and absence of metabolic activation did not show background bacterial lawn inhibition compared to vehicle control groups. There was no dose-related increase in the number of revertant colonies observed in the tester strains treated with test item, both in the presence and absence of metabolic activation. In S9 efficiency check, the number of increasing revertant colonies was not observed in positive 
control plates of 2-aminoanthracene in absence of metabolic activation when compared with presence of metabolic activation, which indicates efficiency of S9 fraction.

In all the tester strains, the frequency of the spontaneous revertant colonies in vehicle control was within the acceptable range of historical control data. The positive controls used in the study exhibited significant increase in the mean number of revertant frequency respective to their strains when compared to vehicle control, indicating the sensitivity of the specific mutagens and confirmed function of metabolic activation system.

Micronucleus assay is the most reliable in vivo assay to assess the induction of chromosomal aberration which is main endpoint of mutagenicity hazard identification and risk assessment ${ }^{23}$. Boswellia serrata treated group when compared to concurrent vehicle control group in both DRF study and main study didn't show statistically significant increase in number of micronucleus. Whereas statistically significant increase in number of micronucleus was observed in positive control group.

In single dose acute oral toxicity study, it was found that up to $2000 \mathrm{mg} / \mathrm{kg}$ body weight of Boswellia serrata extract didn't show any morbidity and mortality.

In 90 day repeated dose toxicity study, all hematological parameters in animals of different treated groups of both the sex were comparable to control group, except statistically significant change was observed in Neutrophils, Lymphocyte, aPTT, MCHC, Platelets, Eosinophils and Monocytes in different dose treated group when compared to control group of main study and recovery groups. The observed variations in hematological parameters were considered to be of no toxicological significance and a minimal in nature and occurred in the absence of clear dose related response.

At the end of treatment and recovery period, statistically significant change was observed in parameters like Total Protein, Chloride, Potassium, Sodium, ALP,GGT and Creatinine kinase in few animals of different dose group which was considered as an incidental and without clear dose related distribution in either male or female rats. These alterations have no biological impact on the individual animal and further it is not evidenced by variations in organ weight and histopathological observations. Hence it could be considered as an incidental finding and not incurred due to administration of the test item.

There were no treatment-related changes in urine parameters in both the sexes of treated and treatment free groups at the end of treatment and recovery period. All of the physical, biochemical and microscopic parameters of urine in treated rats were well comparable to control group rats. External examination of all male and female rats of control and all treated groups did not reveal any abnormality of pathological significance. Visceral examination of the rats of control and other treated groups did not reveal any pathological abnormality.

Absolute and relative weight of organs of treated group rats of either sex did not differ significantly when compared to the respective control group ratsat the end of treatment period. Few minor variations without clear dose related distribution was observed viz., statistically significant decrease were observed in absolute splenic weight in male rats treated at $60 \mathrm{mg} / \mathrm{kg}$ as compared to control group, statistically significant increased were noted in relative weight of liver treated at $120,240 \mathrm{mg} / \mathrm{kg}$ in females as compare to control group in both main and recovery group animals.

External examination of all male and female rats from control and all treated groups including recovery groups did not reveal any abnormality of pathological significance. Visceral examination of the rats from control and other treated groups did not reveal any pathological abnormality except size reduction in testes was observed in one male rat of G4R group.

Microscopic examination of control group and rats treated at $240 \mathrm{mg} / \mathrm{kg}$ revealed varying degree of pathological changes in different organs. Lesions observed in liver, kidneys, lungs, aorta, heart, colon, thymus, trachea, adrenal gland, skin of high dose treated group are well comparable with respective control group rats and exhibited no dose relationship. Greaves, 2007 opined that laboratory animals develop testicular atrophy spontaneously and it may be seen sporadically, therefore, observed atrophic lesion of testis and epididymides can be considered as spontaneous. All the observed lesions are minimal to mild in nature. Further these observed lesions are commonly observed in rodents during toxicological studies ${ }^{24-26}$. Hence, occurrence of these lesions could be considered as spontaneous or incidental in nature. 


\section{Conclusion}

Our result shows that Boswellia serrata extract is non-mutagenic from Bacterial reverse phase mutation assay, bone marrow chromosomal aberration test and bone marrow micronucleus test. Single dose acute oral toxicity study up to $2000 \mathrm{mg} / \mathrm{kg}$ body weight of Boswellia serrata extract didn't show any mortality. 90 days subchronic study of Boswellia serrata extract up to the dose of $600 \mathrm{mg} / \mathrm{kg}$ did not show any adverse effect and can be concluded that $600 \mathrm{mg} / \mathrm{kg}$ repeated dose in the experimental animals is safe and can be considered as "No Observed Adverse Effect Level" (NOAEL) under the experimental condition applied. Thus, Boswelliaserrata extract remains unclassified under the hazard category as per Globally Harmonized Classification System (GHS).

\section{REFERENCES}

[1] Sharma, S.; Thawani, V.; Hingorani, L.; Shrivastava, M.; Bhate, V. R.; Khiyani, R., Pharmacokinetic study of 11-Keto betaBoswellic acid. Phytomedicine 2004,11 (2-3), 255-60.

[2] Siddiqui, M., Boswellia serrata, a potential antiinflammatory agent: an overview. Indian journal of pharmaceutical sciences 2011,73 (3), 255.

[3] Raffaelli, M.; Mosti, S.; Tardelli, M., Boswellia sacra Flueck.(Burseraceae) in the Hasik area (Eastern Dhofar, Oman) and a list of the surrounding flora. Webbia 2006,61(2),245-251.

[4] Bhargava, G.; Negi, J.; Guha, S., Studies on the chemical composition of salai gum. Indian Forester 1978,104 (3), 174-181.

[5] Al-Harrasi, A.; Rehman, N. U.; Khan, A. L.; Al-Broumi, M.; Al-Amri, I.; Hussain, J.; Hussain, H.; Csuk, R., Chemical, molecular and structural studies of Boswellia species: $\beta$ Boswellic Aldehyde and 3-epi-11 $\beta$-Dihydroxy BA as precursors in biosynthesis of boswellic acids. PloS one 2018,13 (6).

[6] Singh, G.; Atal, C., Pharmacology of an extract of salai guggal ex-Boswellia serrata, a new non-steroidal anti-inflammatory agent. Agents and Actions 1986,18 (3-4), 407-412.

[7] Sharma, M.; Bani, S.; Singh, G., Anti-arthritic activity of boswellic acids in bovine serum albumin (BSA)-induced arthritis. International Journal of Immunopharmacology 1989,11 (6), 647-652.

[8] Singh, G.; Singh, S.; Bani, S., Boswellic Acids. Antiinflammatory, Antiarthritic, Antipyretic. Drugs of the Future 1993,18, 307-307.

[9] Singh, G.; Singh, S.; Bani, S., Boswellic acids a new anti arthritic agent. Annual Drug data report. 1989, 11, 439-440.
[10] Zhang, Y.; Ning, Z.; Lu, C.; Zhao, S.; Wang, J.; Liu, B.; Xu, X.; Liu, Y., Triterpenoid resinous metabolites from the genus Boswellia: pharmacological activities and potential species-identifying properties. Chemistry Central Journal 2013,7 (1), 153.

[11] Abdel-Tawab, M.; Werz, O.; SchubertZsilavecz, M., Boswellia serrata. Clinical pharmacokinetics 2011,50 (6), 349-369.

[12] Sharma, A.; Chhikara, S.; Ghodekar, S.; Bhatia, S.; Kharya, M.; Gajbhiye, V.; Mann, A.; Namdeo, A.; Mahadik, K., Phytochemical and pharmacological investigations on Boswellia serrata. Pharmacognosy Reviews2009, 3(5), 206.

[13] Hoffmann, G. R., Mutagenicity testing in environmental toxicology. Environmental science \& technology 1982,16 (10), 560A-574A.

[14] Guideline, O., Test No. 423: Acute Oral Toxicity-Acute Toxic Class Method. 2002.

[15] Co-operation, O. f. E.; Development, Test No. 408: Repeated Dose 90-Day Oral Toxicity Study in Rodents. OECD Publishing: 1998.

[16] Guideline, O. T., 475: Mammalian bone marrow chromosomal aberration test. Paris: Organization for Economic Cooperation and Development1997.

[17] OECD, Test No. 487: In vitro mammalian cell micronucleus test. OECD Guidelines for Testing of Chemicals Section for Health Effects 2014.

[18] OECD, T. N., 471: Bacterial reverse mutation test. OECD Guidelines for the Testing of Chemicals, Section 1997,4.

[19] Maron, D. M.; Ames, B. N., Revised methods for the Salmonella mutagenicity test. Mutation Research/Environmental Mutagenesis and Related Subjects 1983,113 (3-4), 173-215.

[20] Qurishi, Y.; Hamid, A.; Zargar, M.; Singh, S. K.; Saxena, A. K., Potential role of natural molecules in health and disease: Importance of boswellic acid. J Med Plants Res 2010,4 (25), 2778-2785.

[21] Kannana, T. P.; Lahc, N.; Ahmada, A.; Fatimah, S., Genotoxic evaluation of synthetic hydroxyapatite using mammalian bone marrow chromosome aberration test. Arch Orofac Sci 2014,9 (1), 1-7.

[22] Demma, J.; Engidawork, E.; Hellman, B., Potential genotoxicity of plant extracts used in Ethiopian traditional medicine. Journal of Ethnopharmacology 2009,122 (1), 136-142.

[23] Hayashi, M., The micronucleus test - most widely used in vivo genotoxicity test- Genes and Environment 2016,38 (1), 18.

[24] Greaves, H., Probability in the Everett interpretation. Philosophy Compass 2007,2 (1), 109-128. 
Mutagenic, Genotoxic and Sub Chronic Oral Safety Analysis of Boswellia Serrata Extract (Boswellin ${ }^{\circledR}$ Super)

[25] Greaves, P., Histopathology of preclinical toxicity studies: interpretation and relevance in drug safety evaluation. Academic Press: 2011.
[26] Haschek, W.; Voss, K.; Beasley, V., Selected mycotoxins affecting animal and human health. Handbook Toxicol. Pathol., 1: 645 2002,699.

Citation: Muhammed Majeed et al., "Mutagenic, Genotoxic and Sub Chronic Oral Safety Analysis of Boswellia Serrata Extract (Boswellin ${ }^{\circledR}$ Super)”, International Journal of Research Studies in Medical and Health Sciences. 2020; 5(7): 20-32.

Copyright: (C) 2020 Muhammed Majeed et al., This is an open-access article distributed under the terms of the Creative Commons Attribution License, which permits unrestricted use, distribution, and reproduction in any medium, provided the original author and source are credited. 